V - 'appetite and weight change', VI - 'anxiety' and VII - 'anergia'. A significant therapeutic effect of lamotrigine in bipolar depression was found using the 'depressive cognitions' factor (from week 3) and 'psychomotor retardation' (from week 4).

Conclusions: This study has identified seven factors of the HDRS in a large sample of patients with bipolar depression. It suggests that the major effect of lamotrigine in bipolar depression is primarily upon central depressive cognitions and psychomotor disturbance.

\section{Evaluation of MoodGYM with an adolescent population}

\section{A Neil, H Christensen, K Griffiths}

Centre for Mental Health Research, The Australian National University, Canberra, Australia

Background: MoodGYM is an interactive web-based program designed to prevent and decrease symptoms of anxiety and depression. Based on cognitive behaviour therapy, the MoodGYM program aims to change dysfunctional thoughts and improve problem solving, relaxation and interpersonal skills. Although much research has been conducted on the effectiveness of MoodGYM with an adult population, with very positive results, little research has been conducted on the adolescent and young adult population for which the program was designed. The current study therefore aims to evaluate the effectiveness of the MoodGYM program in reducing adolescents' symptoms of anxiety and depression.

Methods: Twenty-nine schools from across Australia were involved in the current study, with 1450 students aged 13-17 years consenting to participate. Each school was randomly assigned to the experimental or control group based on their location (city or rural) and funding (government or private). All participating students completed the first self-report questionnaire the week before students in the experimental condition began the MoodGYM program. The MoodGYM program was delivered to students over a 5-week period. All participating students completed a second and third self-report questionnaire 1 and 6 months after the completion of the MoodGYM program.

Results: The current paper will present preliminary results from the first two questionnaires. Pre- and posttest comparisons will be made for the measures of anxiety and depression.

Conclusion: Findings from the current study will help to inform schools about the usefulness of a self-guided approach to learning about mental health using the Internet.

\section{Visual field semantic priming and relationship with thought disorder}

\author{
E Neill', M Collins'², S Collinson ${ }^{3}$, S Rossell'
}

'Mental Health Research Institute of Victoria, Melbourne, Australia; ${ }^{2}$ Murdoch

University, Perth, Australia; and ${ }^{3}$ Singapore University, Singapore

Background: The semantic priming technique can be used to explore language deficits in individuals with thought disorder. Research suggests that thought disorder may reflect a difficulty in inhibition or an increase in spreading activation in one or both of the hemispheres in response to language-based information. Currently, little research has been done in this area and the research that is available is methodologically flawed.

Methods: A lateralized version of a semantic priming task was used to examine the role of each hemisphere in processing language and to examine how the two hemispheres synchronize information. As in standard semantic priming task, reaction times to related and unrelated word pairs were contrasted. Novelly, to this experiment, word pairs were presented solely to the left (LL) or right (RR) visual field, or across visual fields; left-right (LR) or right-left (RL). Participants included 21 healthy controls and 42 participants with a diagnosis of schizophrenia (21 with thought disorder, 21 without). Repeated-measure ANOVAs were used to compare stimulus type (related vs. unrelated), visual field (LL, RR, LR, RL) and group.

Results: Preliminary analyses suggest that there are significant differences in priming across visual fields between healthy controls and participants with schizophrenia. There were no significant differences between the two psychiatric groups.

Conclusions: These data suggest that participants with schizophrenia have difficulty when performing a task where the two hemispheres must synchronize information. However, this pattern this does not seem to be causal for thought disorder.

\section{When things are not as they seem: detecting first-episode psychosis upon referral to ultrahigh-risk ('prodromal') clinics}

\section{B Nelson', A Yung ${ }^{2}$ \\ 'ORYGEN Research Centre; and 'Department of Psychiatry, The University of Melbourne, Melbourne, Australia}

Background: There are two main targets for intervention during the prodromal phase: 1) current symptoms, behaviour or disability and 2) prevention of onset of frank psychotic disorder. The current presentation examines a 'third' function of 'ultrahigh-risk' (UHR) 
clinics: to detect first-episode psychosis (FEP) mistakenly identified as a prodrome.

Methods: A clinical audit was conducted of referrals to a UHR service, the Personal Assessment and Crisis Evaluation (PACE) clinic, over a 12-month period (April 2005 to March 2006) to establish the proportion who were psychotic on referral.

Results: The PACE clinic received 149 referrals over the period examined. About $11.4 \%$ of the total number of referrals and $12.6 \%$ of those who attended a first appointment were psychotic on referral.

Conclusions: These figures indicate that a substantial proportion of individuals thought to be prodromal are in fact suffering FEP. It is suggested that by detecting these individuals and commencing treatment, UHR clinics minimize duration of untreated psychosis for patients with FEP mistaken as prodromal.

\section{Obsessive compulsive disorder: time to decide on a new model?}

\author{
A Olley', G Malhi ${ }^{2,3,4}$, P Sachdev ${ }^{1,5}$ \\ 'School of Psychiatry, University of New South Wales; ${ }^{2}$ Academic Discipline of \\ Psychological Medicine, Northern Clinical School, The University of Sydney; ${ }^{3}$ The \\ Black Dog Institute, Sydney, Australia; 4Prince of Wales Medical Research Institute, \\ Sydney, Australia; and ${ }^{5}$ Neuropsychiatric Institute, Prince of Wales Hospital, \\ Sydney, Australia
}

Background: Obsessive compulsive disorder (OCD) is a chronic and debilitating disorder characterized by chronic doubt and indecision; yet, decision making as a cognitive construct has received little attention in either the neuropsychological or the neuroimaging studies of OCD. Indeed, the neuropsychological profile of OCD remains poorly defined and further investigations are needed. To date, most studies have been exploratory in nature and not based on theoretical frameworks.

Methods: A selective review of memory and executive functioning in OCD was conducted using the MEDLINE database, with particular focus on decision making. In addition, the neurobiology of both OCD and decision making was reviewed.

Results: OCD, although once believed to be a disorder of memory, is now viewed primarily as a disorder of executive functioning, a view which is strongly supported by findings in neuroimaging. Decision making, although little studied, is emerging as a potential area of dysfunction that warrants further investigation.

Conclusions: Given the incapacity that results from chronic doubt and indecision in the clinical population, it is surprising that decision making has not received greater consideration in conceptualizing this disorder. We posit a tentative model of OCD that provides a unique perspective of OCD within a decision-making framework. To examine the utility of this model, we propose a multimodal study incorporating functional neuroimaging and neuropsychological assessment in an attempt to dissect the individual stages of decision making in OCD. Understanding the neuroanatomical substrates will lead to the development of new physical and psychological treatments.

\section{They never forget: recall of traumatic memories of Vietnam}

\section{B 0'Toole', K Pierse', J Cockburn², S Catts ${ }^{3}$ \\ 'ANZAC Research Institute; ' University of Newcastle; and ${ }^{3} U$ niversity of Queensland, Brisbane, Australia}

Background: The establishment of a diagnosis of post-traumatic stress disorder (PTSD) requires first that the person has actually experienced a traumatic event. The second wave of the Australian Vietnam Veterans Health Study allowed us to observe the consistency of reports of war trauma 30 or more years after the trauma and 15 years after their initial recounting.

Methods: Interviews were conducted in 2005-2006 with a cohort of randomly selected Australian Army Vietnam veterans who were first assessed using standardized PTSD diagnostic assessments during 19911993. A standardized scale was used on both occasions to measure war trauma exposure and to act as a cue to eliciting recalled and reported events that would qualify under the DSM ' $A$ ' criterion. One or more distressing events were elicited and transcribed for a qualitative analysis of the war stories offered in 2005-2006 and 1991-1993.

Results: Time 1 to time 2 correlations between each of the 21 items in the combat scale were highly positive, as was the total. The factor structures of the two administrations were very similar, as were item-total correlations and Cronbach's $\alpha$. Qualitative analysis of stories showed that almost identical stories were told on the two occasions when nominating the 'worst' events of Vietnam. However, some of the details emerged over several hours of interviewing, which indicates that veterans may not be as open to disclosing their war histories in short encounters.

Conclusion: Soldiers' memory for war-related trauma is very stable, even 40 years after war's end.

\section{The prevalence of PTSD in Australian Vietnam Veterans 35 years after war's end: preliminary results of a cohort study}

\section{B O'Toole', K Pierse', S Outram², J Cockburn², S Catts ${ }^{3}$}

'ANZAC Research Institute; ${ }^{2}$ University of Newcastle; and ${ }^{3}$ University of Queensland, Brisbane, Australia 